\title{
Robust Distributed Control Design for Interconnected Systems under Topology Uncertainty
}

\author{
Dong Xue, Azwirman Gusrialdi and Sandra Hirche
}

\begin{abstract}
This paper jointly designs the distributed control law and its communication architecture for large-scale interconnected systems. The novelty is to consider both, the robustness guaranty under topology uncertainty and the performance improvement of the overall system. The proposed framework consists of two steps. The first step aims at finding a set of stabilizing distributed control laws providing $H_{\infty}$ robust performance under topology uncertainty. In the second step the performance of the interconnected system is further improved by searching for the optimal communication topology within acquired set of distributed control laws. In this step, the design of communication architecture is achieved by minimizing the $\mathrm{H}_{2}$ norm. Additionally, a trade-off between performance improvement and communication cost is incorporated. Furthermore, to reduce computational complexity, a strategy to manipulate the existing communication links is proposed to deal with topology variation in the interconnected systems. Finally, the developed method is demonstrated via some examples.
\end{abstract}

\section{INTRODUCTION}

Many practical systems in engineering can be modeled as large-scale interconnected systems such as power grids [1], water distribution networks and transportation systems. The design of control algorithms for such interconnected systems has received considerable attention in recent years [2]-[5].

The individual subsystems in a large-scale interconnected system are physically coupled; typically the overall system has a certain sparsity structure. For such large-scale systems, centralized or conventional control methods become infeasible since they assume that a single centralized controller has instantaneous access to all measurements. In order to address this problem, in early works decentralized control schemes are developed, see e.g. [6] for an overview. The fundamental idea is to utilize only locally available state information in designing the control law, while the performance might be significantly degraded compared to a centralized approach. Advances in digital communication technologies allow for communication between subsystems and, thereby, distributed control schemes are facilitated. They provide a larger control flexibility: instead of only local subsystem information, the states of neighboring subsystems can be used for control as well. As a result, a better performance is typically achieved compared to decentralized approaches [7].

One of the challenges in designing distributed control laws is the topology uncertainty due to the physical or communication link failures. Such uncertainty may harm the

D. Xue and S. Hirche are with the Institute for Information-oriented Control, Technische Universität München, Arcisstraße 21, D-80290 München, Germany; dong. xue@tum. de, hirche@tum. de.

A. Gusrialdi is with Dept. Electrical Engineering and Computer Science, University of Central Florida, USA; Azwirman.Gusrialdi@ucf .edu stability and performance of the overall system. For example, a transmission link failure in a power grid may result in a cascading failure which can possibly lead to a large blackout [8]. In addition, it is desirable that external disturbances, for example load or renewable energy resource fluctuation in a smart grid, diminish as they propagate through the system [1]. The robustness to topology uncertainty and disturbance rejection is crucial and in the focus of this paper.

In practical engineering, the controller is expected not to only eliminate the disturbance, but also optimize a desired control performance involved with certain worst-case disturbance. Since $\mathrm{H}_{2}$ performance is very appealing for many applications, $\mathrm{H}_{2}$ optimization is widely applied in optimal control problems, see e.g. [9]. Another issue relevant to interconnected systems is the communication cost for information transmission. In particular, an appropriate trade-off between performance improvement and the number of communication links is desirable. Optimal sparse state feedback control laws are developed in [3], [4], and for output feedback in [5]. However, no topology uncertainty is taken into account.

The main contribution of this paper is to design an optimal distributed control law for large-scale interconnected systems with topology uncertainty. The proposed strategy involves two steps: The first step aims at identifying distributed control gains subject to external disturbance and topology uncertainty for which stability and a certain level of $H_{\infty}$ performance can be guaranteed. This topology uncertainty is characterized by introducing a local connectivity bound to candidate structures. The feedback control gains are derived from the solution of linear matrix inequalities (LMIs). In the second step, the performance is further improved by extracting the optimal communication topology from the family of robust topologies characterized in the first step. This is achieved by minimizing the $H_{2}$ norm from an external disturbance to the controlled output and simultaneously taking into account the trade-off between performance improvement and communication cost. The optimization problem is posed as a mixed-integer semi-definite program (MISDP), which can be solved using, for example, branch-and-bound algorithms. The framework developed in this paper has following advantages: 1) It is not required to redesign the control law when the topology structure or size of system changes as long as a local connectivity bound remains satisfied; 2) the local connectivity constraint provides an explicit rule on regulating the interconnected systems without harming the stability of the overall system; 3 ) it also provides the controller sufficient time to re-optimize its communication topology when the topology changes without losing of stability, which will be 
demonstrated later in the paper.

The organization of this paper is as follows: after formulating the problem in Section II, the control design for $H_{\infty}$ performance is proposed in Section III. In Section IV, based on the previous results, an optimal communication topology is identified. Furthermore, a heuristic manipulation algorithm of communication links is presented. Finally, the proposed strategies are evaluated via numerical examples in Section V. Notation. Let $\mathbb{R}$ be the set of real numbers; $\operatorname{diag}(a, b)$ represents the diagonal matrix $\left[\begin{array}{ll}a & 0 \\ 0 & b\end{array}\right]$, where $a, b \in \mathbb{R} ; \mathbf{1}(\mathbf{0})$ denotes the $N \times 1$ column vector of all ones (zeros), and $I_{N}\left(\mathbf{0}_{N}\right)$ is the $\mathrm{N}$-dimensional identity (zero) matrix (for simplicity $I$ and 0 if no confusion arises). Moreover, let $\{0,1\}_{N}$ be the set of all $N$-dimensional $0-1$ matrix. $\operatorname{tr}(\cdot)$ represents the trace function and the operator $\circ$ is the Hadamard product.

\section{PRoblem Formulation}

Consider an interconnected system of $N$ linear time invariant (LTI) subsystems with dynamics described by the following differential equations

$$
\begin{aligned}
& \dot{x}_{i}=A_{i} x_{i}+\sum_{j=1}^{N} U_{i j} A_{i j} x_{j}+B_{1, i} w_{i}+B_{2, i} u_{i}, \\
& z_{i}=C_{i} x_{i}, \quad i=1,2, \ldots, N
\end{aligned}
$$

where $x_{i} \in \mathbb{R}^{n}, u_{i} \in \mathbb{R}^{n_{u}}$ are the state of subsystem $i$ and the control input to subsystem $i$, and matrices $A_{i}, A_{i j}, B_{1, i}, B_{2, i}$ and $C_{i}$ are real and of compatible dimensions. For notational convenience, we consider the dimension for all subsystems equal but that the approach straightforwardly extends to different dimensions. The performance output $z_{i} \in \mathbb{R}^{n_{z}}$ represents an error signal, and the exogenous signal $w_{i} \in \mathbb{R}^{n_{w}}$ denotes all external inputs, including sensor noise, disturbance, and commands. The term $\sum_{j=1}^{N} U_{i j} A_{i j} x_{j}$ represents the physical coupling with neighboring subsystems, where matrix $A_{i j}$ and scalar $U_{i j} \in\{0,1\}$ are the coupling strength and index between subsystem $i$ and $j$, respectively. The index $U_{i j}$ is equal to 1 when there is a physical connection between subsystem $i$ and $j(j \neq i)$; otherwise 0 . Furthermore, when $j=i$, we have $U_{i i}=0$. In many practical situations, the physical interconnection between any two subsystems may not always be available or fixed due to failure of physical devices, geographical limitation or environment variation. Motivated by recent technological advances on communication topologies, remote non-local information can be used to implement the local control law given by

$$
u_{i}=K_{i} x_{i}+\sum_{j=1}^{N} V_{i j} K_{i j} x_{j}, \quad \forall i=1, \ldots, N,
$$

where the feedback gain $K_{i j}$ and the interconnection index $V_{i j}$ have an analogous interpretation as their counterparts in the physical layer. The interconnected system (1) with its distributed control law (2) is illustrated in Fig.1.

The physical interconnection among the subsystems incorporated with the distributed control, i.e. communication topology, are represented by the joint graph $\mathcal{G}=\left(\mathcal{S}, \mathcal{E}_{U} \cup \mathcal{E}_{V}\right)$, where $\mathcal{S}=\left\{s_{1}, \ldots, s_{N}\right\}$ denotes the

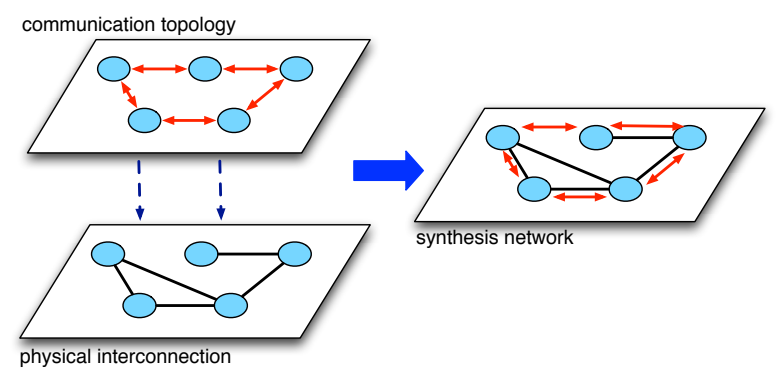

Fig. 1. Synthesis network of physical interconnection and communication topology

set of subsystems, $\mathcal{E}_{U}$ and $\mathcal{E}_{V}$ are the set of edges in the physical and the communication topology. Note that for $\mathcal{E}_{V}=\varnothing$, the control law reduces to a decentralized one. To facilitate the following analysis, we only confine our attention to undirected graphs, i.e. $U_{i j}=U_{j i}$ and $V_{i j}=V_{j i}$.

Remark 2.1: The decomposition into coupling terms $A_{i j}$ in the system dynamics and $K_{i j}$ on the control level and the interconnection topology represented by the adjacency matrices $U=\left[U_{i j}\right]_{N \times N}, V=\left[V_{i j}\right]_{N \times N}$ is introduced to represent topological uncertainties in a convenient fashion. The adjacency matrices $U$ and $V$ represent the interconnection between subsystems, i.e. cluster of states. Also note that the undirected graph assumption $U=U^{\top}$ and $V=V^{\top}$ does not imply that the system dynamics/control law is symmetric; i.e. $A_{i j}$ and $A_{j i}$ are not necessarily equal.

In order to represent topology uncertainty we will use the notion of a degree of a vertex. The degree $d_{i}$ of subsystem $i$ is defined as the number of neighboring subsystems within the joint graph $d_{i}(\mathcal{G})=\sum_{j=1}^{N}\left(1-\left(1-U_{j i}\right)\left(1-V_{j i}\right)\right)$. We assume that the joint graph is connected and there is no isolated subsystem in the interconnected system (1), i.e. $d_{i}>1 \forall i \in\{1, \ldots, N\}$. In practical interconnected systems, this assumption does not represent any restriction for our analysis because a subsystem without any interconnection makes no contribution to the performance of overall system. A local connectivity bound $d$ is introduced to characterize the structural properties of the admissible system topologies within an uncertainty set, where $1<d \leq N-1$. Associated with connectivity bound, we define a set of graphs

$$
\mathbb{G}(d)=\left\{\mathcal{G} \mid d_{i}(\mathcal{G}) \leq d, \forall i=1, \ldots, N, \text { and } \mathcal{G} \text { is connected }\right\},
$$

which corresponds to our definition of topology uncertainty in this paper. Note that $d>1$ is derived from that graph $\mathcal{G}$ is connected, and for $d=N-1$ the set $\mathbb{G}(d)$ includes arbitrary topologies for the given number of vertices. Smaller $d$ reduces the cardinality of the set and puts a stronger restriction on the admissible topologies in this set. It is interesting to note, that a violation of the connectivity bound can be decided locally, i.e. each node can decide whether it satisfies the degree bound without the need of global information.

For further derivations we denote the aggregated state vector for the overall system as $x=\left[x_{1}^{\top}, \ldots, x_{N}^{\top}\right]^{\top}$. The closed loop dynamics of the overall system can then be written as

$$
\dot{x}=\left(\hat{A}+B_{2} \hat{K}\right) x+B_{1} w, \quad z=C x,
$$


where $z=\left[z_{1}^{\top}, \ldots, z_{N}^{\top}\right]^{\top}, \quad B_{1}=\operatorname{diag}\left(B_{1,1}, \ldots, B_{1, N}\right)$, $B_{2}=\operatorname{diag}\left(B_{2,1}, \ldots, B_{2, N}\right), \quad C=\operatorname{diag}\left(C_{1}, \ldots, C_{N}\right)$ and $\hat{A}=A_{\text {ind }}+U \circ A_{\text {int }}$, where $A_{\text {ind }}=\operatorname{diag}\left(A_{1}, \ldots, A_{N}\right)$ and

$$
A_{\text {int }}=\left[\begin{array}{cccc}
0 & A_{12} & \cdots & A_{1 N} \\
A_{21} & 0 & \cdots & A_{2 N} \\
\vdots & \vdots & \ddots & \vdots \\
A_{N 1} & A_{N 2} & \cdots & 0
\end{array}\right]
$$

The controller is analogously decomposed into a local control term and a coupling control term, see also (2), by

$$
\hat{K}=K_{\text {ind }}+V \circ K_{\text {int }},
$$

where $V=\left[V_{i j}\right]_{N \times N}$ is adjacency matrix of the communication topology. Additionally, we assume that $\left(\hat{A}, B_{2}\right)$ is stabilizable and $(\hat{A}, C)$ is detectable.

Now we formally state the problem being investigated in this paper. The goal is to design the distributed feedback gains together with the communication topology in (4) such that: 1) stability is preserved under topology uncertainty and 2) the control performance is guaranteed within a desired level. In order to deal with disturbances and topology uncertainty, we first identify a set of distributed control laws which ensures a certain $H_{\infty}$ performance level for all graphs $\mathcal{G} \in \mathbb{G}(d)$. Next, by the flexibility of allocating the communication links within the set $\mathbb{G}(d)$, the performance is further improved by solving an $\mathrm{H}_{2}$ optimal problem while reducing the communication cost.

\section{Control Design For Topology-Robust $H_{\infty}$ PERFORMANCE}

Denote $T_{z w}$ as the transfer function matrix from the disturbance $w$ to the controlled output $z$ of the interconnected system (3), i.e. $z=T_{z w} w$. The classical $H_{\infty}$ problem can be stated as following: given a desired scalar $\gamma>0$, find an appropriate control protocol $\hat{K}$, such that the system is stable, while $\left\|T_{z w}\right\|_{\infty}<\gamma$. When there are no constraints on the structure of $\hat{K}$, by the Kalman-Yakubovich-Popov lemma [10], satisfying the $H_{\infty}$ norm $\left\|T_{z w}\right\|_{\infty}<\gamma$ implies that there exists a matrix $\mathcal{P} \succ 0$ such that Riccati inequality $\left(\hat{A}+B_{2} \hat{K}\right) \mathcal{P}+\mathcal{P}\left(\hat{A}+B_{2} \hat{K}\right)^{\top}+B_{1} B_{1}^{\top}+\frac{1}{\gamma^{2}} \mathcal{P} C^{\top} C \mathcal{P} \prec 0$ holds. In order to simplify the analysis and to render the problem computationally tractable, we assume that the $\mathcal{P}$ are restricted to diagonal form $\mathcal{P}=\operatorname{diag}\left(P_{1}, \ldots, P_{N}\right)$, where $P_{i} \succ 0$ for $i=1, \ldots, N$. This diagonal design on $\mathcal{P}$ introduces some conservatism, i.e. the feasible $H_{\infty}$ performance will present an upper bound only within the uncertainty set $\mathbb{G}(d)$ of admissible topologies.

To obtain the desired form of $\hat{K}$, a new variable $Q=\hat{K} \mathcal{P}$ is introduced and presented in the form

$$
\begin{aligned}
Q & =Q_{\text {ind }}+V \circ Q_{\text {int }}=\operatorname{diag}\left(Q_{\text {ind }}^{1}, \ldots, Q_{\text {ind }}^{N}\right) \\
& +\left[\begin{array}{cccc}
0 & V_{12} & \cdots & V_{1 N} \\
V_{21} & 0 & \cdots & V_{2 N} \\
\vdots & \vdots & \ddots & \vdots \\
V_{N 1} & V_{N 2} & \cdots & 0
\end{array}\right] \circ\left[\begin{array}{cccc}
0 & Q_{\text {int }}^{12} & \cdots & Q_{\text {int }}^{1 N} \\
Q_{\text {int }}^{21} & 0 & \cdots & Q_{\text {int }}^{2 N} \\
\vdots & \vdots & \ddots & \vdots \\
Q_{\text {int }}^{N 1} & Q_{\text {int }}^{N 2} & \cdots & 0
\end{array}\right]
\end{aligned}
$$

where $Q_{\text {ind }}^{i}=K_{i} P_{i}$ and $Q_{\text {int }}^{i j}=K_{i j} P_{j}$. The Riccati inequality can then be rewritten as

$$
\hat{A} \mathcal{P}+\mathcal{P} \hat{A}^{\top}+B_{2} Q+Q^{\top} B_{2}^{\top}+B_{1} B_{1}^{\top}+\frac{1}{\gamma^{2}} \mathcal{P} C^{\top} C \mathcal{P} \prec 0 .
$$

Under the restriction on $\mathcal{P}$, the $i, j$ block of (5) is given by

$$
\begin{aligned}
& \text { for } i=j: A_{i} P_{i}+P_{i} A_{i}^{\top}+B_{2, i} Q_{\text {ind }}^{i}+\left(Q_{\text {ind }}^{i}\right)^{\top} B_{2, i}^{\top}+B_{1, i} B_{1, i}^{\top} \\
& +\frac{1}{\gamma^{2}} P_{i} C_{i}^{\top} C_{i} P_{i} \\
& \text { for } i \neq j: U_{i j} A_{i j} P_{j}+U_{j i} P_{i} A_{j i}^{\top}+V_{i j} B_{2, i} Q_{\text {int }}^{i j}+V_{j i}\left(Q_{\text {int }}^{j i}\right)^{\top} B_{2, j}^{\top} \text {. }
\end{aligned}
$$

In the following analysis, a distributed control law is derived which guarantees that the interconnected system has a robust $H_{\infty}$ performance for any graph $\mathcal{G} \in \mathbb{G}(d)$.

Before stating the main theorem in this paper, we recall the following Lemma.

Lemma 3.1: [11] Let a Hermitian matrix $\Lambda$ be partitioned into blocks $\Lambda_{i j}$, where $i, j=1, \ldots, N$. Suppose the number of nonzero off-diagonal blocks in $i$ th row of $\Lambda$ is $m_{i}$. Without loss of generality, there exist at least one nonzero offdiagonal block in each row. If

$$
\left[\begin{array}{cc}
\frac{1}{m_{i}} \Lambda_{i i} & \Lambda_{i j} \\
\Lambda_{j i} & \frac{1}{m_{j}} \Lambda_{j j}
\end{array}\right] \succ 0
$$

holds for all $i, j=1, \ldots, N, i \neq j$, then $\Lambda \succ 0$.

Theorem 3.1: Consider the interconnected system (3) and let the uncertainty set $\mathbb{G}(d)$ of admissible topologies be parameterized by a degree upper bound $1<d \leq N-1$. If $P_{i} \succ 0, Q_{\text {ind }}^{i}, Q_{\text {int }}^{i j}(i, j=1, \ldots, N)$ are the solutions of the following linear matrix inequalities:

$$
\begin{aligned}
& \bar{A}_{i j} \hat{P}_{i j}+\hat{P}_{i j} \bar{A}_{i j}^{\top}+B_{i j} \bar{Q}_{i j}+\bar{Q}_{i j}^{\top} B_{i j}^{\top}+\hat{B}_{i j} \hat{B}_{i j}^{\top}+\frac{\hat{P}_{i j} C_{i j}^{\top} C_{i j} \hat{P}_{i j}}{\gamma^{2}} \prec 0 \\
& \bar{A}_{i j} \hat{P}_{i j}+\hat{P}_{i j} \bar{A}_{i j}^{\top}+B_{i j} \hat{Q}_{i j}+\hat{Q}_{i j}^{\top} B_{i j}^{\top}+\hat{B}_{i j} \hat{B}_{i j}^{\top}+\frac{\hat{P}_{i j} C_{i j}^{\top} C_{i j} \hat{P}_{i j}}{\gamma^{2}} \prec 0 \\
& \tilde{A}_{i j} \hat{P}_{i j}+\hat{P}_{i j} \tilde{A}_{i j}^{\top}+B_{i j} \bar{Q}_{i j}+\bar{Q}_{i j}^{\top} B_{i j}^{\top}+\hat{B}_{i j} \hat{B}_{i j}^{\top}+\frac{\hat{P}_{i j} C_{i j}^{\top} C_{i j} \hat{P}_{i j}}{\gamma^{2}} \prec 0
\end{aligned}
$$

with the performance level $\gamma>0$ and

$$
\begin{array}{rlrl}
\tilde{A}_{i j}=\left[\begin{array}{cc}
A_{i} & 0 \\
0 & A_{j}
\end{array}\right] & \bar{A}_{i j}=\left[\begin{array}{cc}
A_{i} & d A_{i j} \\
d A_{j i} & A_{j}
\end{array}\right] & \hat{B}_{i j}=\left[\begin{array}{cc}
B_{1, i} & 0 \\
0 & B_{1, j}
\end{array}\right] \\
\hat{P}_{i j}=\left[\begin{array}{cc}
P_{i} & 0 \\
0 & P_{j}
\end{array}\right] & \bar{Q}_{i j}=\left[\begin{array}{cc}
Q_{\text {ind }}^{i} & d Q_{\text {int }}^{i j} \\
d Q_{\text {int }}^{j i} & Q_{\text {ind }}^{j}
\end{array}\right] & B_{i j}=\left[\begin{array}{cc}
B_{2, i} & 0 \\
0 & B_{2, j}
\end{array}\right] \\
C_{i j}=\left[\begin{array}{cc}
C_{i} & 0 \\
0 & C_{j}
\end{array}\right] & \hat{Q}_{i j}=\left[\begin{array}{cc}
Q_{\text {ind }}^{i} & 0 \\
0 & Q_{\text {ind }}^{j}
\end{array}\right],
\end{array}
$$

then the distributed control law (2) with $K_{i}=Q_{\text {ind }}^{i} P_{i}^{-1}$, and $K_{i j}=Q_{\text {int }}^{i j} P_{j}^{-1}$ stabilizes the system (3) and guarantees $\left\|T_{z w}\right\|_{\infty}<\gamma$, for all $\mathcal{G} \in \mathbb{G}(d)$.

Proof: If the three matrix inequalities in the Theorem 3.1 are satisfied for all $i, j=1, \ldots, N$, then it implies that

$$
\left[\begin{array}{cc}
\frac{1}{d} \Phi_{i} & \beta_{k}^{\top} \theta_{i j} \\
\beta_{k}^{\top} \theta_{j i} & \frac{1}{d} \Phi_{j}
\end{array}\right] \prec 0, \quad k=1,2,3
$$


where

$$
\begin{gathered}
\beta_{1}=\left[\begin{array}{l}
\mathbf{1} \\
\mathbf{1} \\
\mathbf{1} \\
\mathbf{1}
\end{array}\right], \quad \beta_{2}=\left[\begin{array}{l}
\mathbf{1} \\
\mathbf{1} \\
\mathbf{0} \\
\mathbf{0}
\end{array}\right], \quad \beta_{3}=\left[\begin{array}{l}
\mathbf{0} \\
\mathbf{0} \\
\mathbf{1} \\
\mathbf{1}
\end{array}\right], \quad \theta_{i j}=\left[\begin{array}{c}
A_{i j} P_{j} \\
P_{i} A_{j i}^{\top} \\
B_{2, i} Q_{\mathrm{int}}^{i j} \\
\left(Q_{\mathrm{int}}^{j i}\right)^{\top} B_{2, j}^{\top}
\end{array}\right], \\
\Phi_{i}=A_{i} P_{i}+P_{i} A_{i}^{\top}+B_{2, i} Q_{\mathrm{ind}}^{i}+\left(Q_{\mathrm{ind}}^{i}\right)^{\top} B_{2, i}^{\top}+B_{1, i} B_{1, i}^{\top}+\frac{P_{i} C_{i}^{\top} C_{i} P_{i}}{\gamma^{2}} .
\end{gathered}
$$

We denote a vector $H_{i j}=\left[U_{i j}, U_{j i}, V_{i j}, V_{j i}\right]^{\top}$. According to Lemma 3.1 and using structure properties $d_{i} \leq d$ within uncertainty set $\mathbb{G}(d)$, if the following linear matrix inequalities

$$
\left[\begin{array}{cc}
\frac{1}{d_{i}} \Phi_{i} & H_{i j}^{\top} \theta_{i j} \\
H_{j i}^{\top} \theta_{j i} & \frac{1}{d_{j}} \Phi_{j}
\end{array}\right] \prec 0, \quad i \neq j,
$$

holds for all $i, j=1, \ldots, N$, then the inequality (5) is achieved. To verify inequality (9), all possible combined structures of topologies are investigated as follows:
1). $U_{i j}=U_{j i}=V_{i j}=V_{j i}=1$;
2). $U_{i j}=U_{j i}=V_{i j}=V_{j i}=0$;
3). $U_{i j}=U_{j i}=1, V_{i j}=V_{j i}=0$;
4). $U_{i j}=U_{j i}=0, V_{i j}=V_{j i}=1$.

The case 1) and 2) can be easily confirmed by inequality (8) with $\beta_{1}$. The inequalities (8) with $\beta_{2}$ and $\beta_{3}$ can be used to verify the case 3 ) and 4 ), respectively. Hence, the inequality (9) holds for all $i \neq j$. Finally, the interconnected system is stable, while $\left\|T_{z w}\right\|<\gamma$ for all graph topologies $\mathcal{G} \in \mathbb{G}(d)$. This completes the proof.

For convenience, we denote a set $\mathbb{V} \subset\{0,1\}_{N}$ to embody all candidate adjacency matrix of $N$ subsystems and whose size is $2^{N(N-1) / 2}$. A constrained subset $\mathcal{V} \subset \mathbb{V}$ given by

$$
\mathcal{V}=\{V \in \mathbb{V} \mid \mathcal{G} \in \mathbb{G}(d)\} .
$$

It is worth mentioning that the outcome of Theorem 3.1 is a set of control laws $\mathcal{K}$, which can be represented by

$$
\begin{array}{r}
\mathcal{K}=\left\{\hat{K} \mid \hat{K} \text { is defined in (4), with } K_{\text {ind }}, K_{\text {int }}\right. \\
\text { derived from Theorem 3.1, } \forall V \in \mathcal{V}\}
\end{array}
$$

In the next section, we determine the best $V$ from set $\mathcal{V}$ in the sense of a $\mathrm{H}_{2}$ criterion. Furthermore, the results in Theorem 3.1 offer some interesting insight into the manipulation of the large-scale interconnected systems. Without violating the local degree constraints, we are allowed to flexibly add or remove communication links. The link gains can be independently calculated in a distributed manner from the LMIs (7). This is very appealing for practical systems, where variations in the topology and also the number of subsystem occur, while privacy and complexity concerns prevent the centralized computation of the control law. One has to pay for these advantages with a certain conservatism in the control design.

\section{Communication Topology Design}

This section is devoted to derive the optimal communication architecture for the interconnected system within the range of the set $\mathcal{V}$ in terms of a $H_{2}$ performance criterion. In addition, a manipulation strategy of communication topology based on the existing communication links is proposed to manage the failure or addition of links in physical networks.

\section{A. Optimal $\mathrm{H}_{2}$ Communication Topology Design}

Within the scope of optimal control design for the interconnected systems, the identification of the favorable interconnection structure under a given performance level is desirable. In contrast to $H_{\infty}$, the $H_{2}$ norm control is more appealing for control engineers to achieve a desired control performance. An optimal $H_{2}$ performance with a guaranteed worst case performance in an $H_{\infty}$ sense (as derived in Section III) is considered here, which leads to the a sub-optimal strategy of allocating the communication links. Moreover, a trade-off between the performance improvement and communication cost is incorporated into the topology design.

The cost function of $\mathrm{H}_{2}$ optimal problem is given by

$$
J(V)=\operatorname{tr}\left(\int_{0}^{\infty} B_{1}^{\top} e^{\left(\hat{A}+B_{2} \hat{K}(V)\right)^{\top} t} C^{\top} C e^{\left(\hat{A}+B_{2} \hat{K}(V)\right) t} B_{1} d t\right)
$$

where the adjacency matrix $V \in \mathcal{V}$ is considered the only decision variable in the cost function. The entries $V_{i j}$ in $V$ are implicitly dependent on connectivity degree $d$ and physical coupling index $U_{i j}$. According to the Theorem 3.1, the feedback controller $u=\hat{K} x$ with $\hat{K} \in \mathcal{K}$ stabilizes the system, i.e. the above integral is bounded and it can be evaluated by solving the Lyapunov equation

$$
\begin{array}{r}
\left(\hat{A}+B_{2}\left(K_{\text {int }}+V \circ K_{\text {ind }}\right)\right)^{\top} \mathcal{Q}+\mathcal{Q}\left(\hat{A}+B_{2}\left(K_{\text {int }}\right.\right. \\
\left.\left.+V \circ K_{\text {ind }}\right)\right)=-C^{\top} C,
\end{array}
$$

where matrix $\mathcal{Q}$ is the observability Gramian of the overall system. As a result, the cost function can be rewritten here as

$$
J(V)=\operatorname{tr}\left(B_{1}^{\top} \mathcal{Q}(V) B_{1}\right)
$$

With respect to the constraint on the number of communication links, a penalty term may also be explicitly incorporated into the objective function as

$$
J(V)=\operatorname{tr}\left(B_{1}^{\top} \mathcal{Q}(V) B_{1}\right)+\frac{\rho}{2} \mathbf{1}^{\top} V \mathbf{1},
$$

where $\rho$ weights the tradeoff of above two terms, with the property that a larger $\rho$ encourages less communication links. It is worth mentioning that when $\rho$ increases large, the distributed control law tends to the decentralized case. However, such decentralization is at the expense of sacrificing the system performance.

Based on above analysis, the network design can be formulated as the following optimization problem:

$$
\begin{array}{cl}
\min _{V_{i j} \in\{0,1\}} & \operatorname{tr}\left(B_{1}^{\top} \mathcal{Q}(V) B_{1}\right)+\frac{\rho}{2} \mathbf{1}^{\top} V \mathbf{1} \\
\text { s.t. } & V=\left[V_{i j}\right]_{N \times N} \in \mathcal{V} \quad \& \quad \text { Eq. (10). }
\end{array}
$$

where $\mathcal{Q}(V)$ is the solution of (10).

The $\mathrm{H}_{2}$ optimal problem incorporated with communication constraints in (12) results in a combinatorial problem. Albeit the existence of nonconvex constraints, numerical software can still be employed to achieve the locally optimal solution. 
Since the undirected graph is taken into account here, the degree constraints in set $\mathbb{G}(d)$ imply that

$$
1<\sum_{j=1}^{N}\left(1-\left(1-U_{i j}\right)\left(1-V_{i j}\right)\right) \leq d, i, j \in\{1, \ldots, N\} .
$$

Let the degree of each subsystem in physical and communication topology respectively be $d_{i}^{p}=\sum_{j=1}^{N} U_{i j}$ and $d_{i}^{c}=\sum_{j=1}^{N} V_{i j}$ and the corresponding degree matrix $D^{p}=\operatorname{diag}\left(d_{1}^{p}, \ldots, d_{N}^{p}\right)$ and $D^{c}=\operatorname{diag}\left(d_{1}^{c}, \ldots, d_{N}^{c}\right)$, respectively. By denoting a matrix $\xi$ with the form $\xi=D^{p}+D^{c}-I \circ(U V)$, the inequality (13) can be rewritten into a compacted matrix inequality as $I \prec \xi \preceq d I$. As a result, the topology design can be reformulated as the following minimization problem.

\section{Optimization Program. I :}

$$
\begin{array}{ll}
\min _{V} & \operatorname{tr}\left(B_{1}^{\top} \mathcal{Q}(V) B_{1}\right)+\frac{\rho}{2} \mathbf{1}^{\top} V \mathbf{1} \\
\text { s.t. } & I \prec \xi(V) \preceq d I \quad \& \quad \text { Eq. (10) }
\end{array}
$$

By Theorem 3.1, if a distributed control law $\hat{K} \in \mathcal{K}$, the $H_{\infty}$ performance is preserved that implies the inverse matrix of $\left(\hat{A}+B_{2} \hat{K}\right)$ exists. Right multiplying $\left(\hat{A}+B_{2} \hat{K}\right)^{-1}$ at both sides, the Lyapunov equation (10) becomes:

$$
\left(\hat{A}+B_{2} \hat{K}\right)^{\top} \mathcal{Q}\left(\hat{A}+B_{2} \hat{K}\right)^{-1}+\mathcal{Q}=-C^{\top} C\left(\hat{A}+B_{2} \hat{K}\right)^{-1} .
$$

Using the cyclic permutations of trace function, the following equation can be obtained from (15)

$$
\operatorname{tr}(\mathcal{Q})=-\frac{1}{2} \operatorname{tr}\left(C^{\top} C\left(\hat{A}+B_{2} \hat{K}\right)^{-1}\right) .
$$

In particular, we discuss the case when the weighted matrix of disturbance $B_{1}$ is identity matrix. Due to the property $\hat{A}+B_{2} \hat{K} \succ 0 \forall \hat{K} \in \mathcal{K}$ and Schur complement, the Optimization Program. I transforms to the following MISDP, which can be solved using a mature and efficient numerical algorithm, for example, branch-and-bound method [12].

\section{Optimization Program. II :}

$$
\begin{array}{ll}
\min _{V} & \operatorname{tr}(F)+\rho \mathbf{1}^{\top} V \mathbf{1} \\
\text { s.t. } & {\left[\begin{array}{cc}
F & C^{\top} \\
C & \hat{A}+B_{2} \hat{K}(V)
\end{array}\right] \preceq 0 \quad \& \quad I \prec \xi \preceq d I .}
\end{array}
$$

\section{B. Topology Manipulation with Existing Links}

In reality, the topology of the interconnected system may change due to physical interconnection failures (e.g. transmission line failures in power system) or some subsystems join in or are removed from the system. Using the proposed approach in Section III, the stability of the interconnected system is preserved under topology variation provided that the new topology belongs to graph set $\mathbb{G}(d)$. However, the performance level may be lost. Compared to redesign the communication topology from Optimization Program. I, the link manipulation based on existing links is more desirable because of less expensive computation and easier implementation. By solving a suboptimal $H_{2}$ problem, we propose a manipulation algorithm based on the optimal solution generated in (14) when the topologies change.
The goal is to reduce the degradation of performance and keep computational complexity low by means of reallocating several existing links. The proposed framework developed in Section III ensures the stability of overall system together with certain worst-case performance, whereby, the communication links can be re-allocated flexibly rather than examining the system performance at each time step.

Let $V^{*}$ be the minimizer derived from Optimization Program. I, which contains $m_{c}$ communication links. The rearrangement of existing links under topology variation can be formulated as following suboptimal problem.

\section{Optimization Program.III :}

$$
\begin{array}{ll}
\min _{V} & \operatorname{tr}\left(B_{1}^{\top} \mathcal{Q}(V) B_{1}\right) \\
\text { s.t. } & \text { Eq. }(10) \& I \prec \xi \preceq d I \& \frac{1}{2} \mathbf{1}^{\top} V \mathbf{1}=m_{c}
\end{array}
$$

Compared with the original optimal problem (14), the solution in the above optimization can be achieved over a set of maximal size $\left(\begin{array}{c}m_{c} \\ N(N-1) / 2\end{array}\right)$ compared with solving the original problem. It should be noted that this topology manipulation given by (17) reduces computational effort while sacrifices partial performance rather than recomputing (14). However, when the scale of systems get larger this design is more appealing, especially, in many practical situations. Furthermore, as stated in [13], the first added links lead to a greater improvement on the performance of overall systems than those added later. That is, the performance is enhanced slightly by adding new links after a certain amount of communication links and subsequently the resulting performance loss is acceptable.

\section{NUMERICAL EXAMPLE}

We consider the system consisting of 5 scalar subsystems with undirected physical interconnections depicted by the black solid lines in Fig.2, and the dynamics are given by $B_{1}=I, B_{2}=I / 3, C=\operatorname{diag}(1.6,0.8,2.4,3.2,1.6)$, and $A_{\text {ind }}=\operatorname{diag}(-2,-2,-4,-3,-2)$

$$
A_{\text {int }}=\left[\begin{array}{ccccc}
0 & 1 & 1 & 2 & 1 \\
0.5 & 0 & 1 & 1.5 & 0.5 \\
1 & 1 & 0 & 1.5 & 0.5 \\
1.5 & 1 & 1.5 & 0 & 0.5 \\
1 & 0.5 & 1 & 0.5 & 0
\end{array}\right]
$$

In order to evaluate the $H_{\infty}$ performance, let $\gamma=0.5$ in this case. The LMIs in (7) with degree constraint $d=2$ can be solved by the YALMIP toolbox [14] and SDPT3 toolbox [15] which result in $K_{\text {ind }}=\operatorname{diag}(-73.1,-35,-101.2,-145.4,-58.6)$ and

$$
K_{\text {int }}=\left[\begin{array}{ccccc}
0 & -0.9 & -1.8 & -3.7 & -1.4 \\
-1.8 & 0 & -2.8 & -3.9 & -1.0 \\
-1.3 & -1.0 & 0 & -2.7 & -0.8 \\
-1.9 & -1.0 & -2.0 & 0 & -0.5 \\
-1.7 & -0.6 & -1.4 & -1.3 & 0
\end{array}\right]
$$

The control gain obtained from LMI leads to an upper bound on the $H_{\infty}$ performance of interconnected systems for 


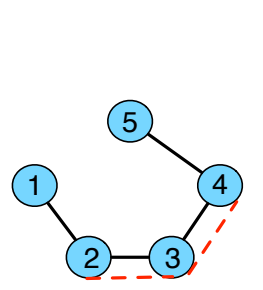

(a)

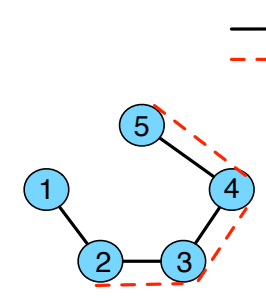

(b)
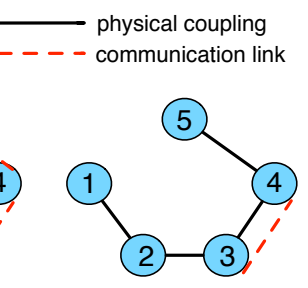

(c)
Fig. 2. synthesis interconnection with different communication penalty: (a). $\rho=7 \times 10^{-5}$; (b). $\rho=5 \times 10^{-5}$; (c). $\rho=1 \times 10^{-4}$

TABLE I

TRADE-OFF BETWEEN $H_{2}$ PERFORMANCE AND COMMUNICATION COST

\begin{tabular}{|c||c||c||}
\hline$\rho$ & Communication Links & $\mathrm{H}_{2}$ norm \\
\hline $3 \times 10^{-5}$ & $(2,3),(3,4),(4,5)$ & 0.3977 \\
\hline $5 \times 10^{-5}$ & $(2,3),(3,4)$ & 0.3978 \\
\hline $1.2 \times 10^{-4}$ & $(3,4)$ & 0.3979 \\
\hline
\end{tabular}

any graph $\mathcal{G} \in \mathbb{G}(d)$. Next, the communication topology is determined by solving the $\mathrm{H}_{2}$ optimal problem presented in (16) w.r.t. penalty weight $\rho=5 \times 10^{-5}$. As a result, communication topology is shown by red dash lines in Fig.2(a) and the corresponding $H_{2}$ norm equals to 0.3978 .

In addition, the tradeoff between the achievable performance and the required communication cost is investigated whose results are summarized in Table I for different $\rho$ values. As shown in Table I, utilizing more communication links results in a smaller $\mathrm{H}_{2}$ performance but at the price of higher communication cost. Fig.2. shows the corresponding communication architectures.

Based on above result, the following two scenarios are further considered to illustrate the manipulation algorithm: 1). addition of a new physical link $(1,5) ; 2)$. deletion of the link $(1,2)$. The solutions using algorithm (17) are explicitly shown in Fig. 3. Compared with the results from solving Optimization Program I for these two cases, which respectively need an additional link $(2,3)$ and link $(4,5)$, the performance degradation of topology manipulation is $0.05 \%$ and $0.03 \%$. This loss rate of performance with lower complexity may become appealing as the scale of the system gets larger.

\section{CONCLUSIONS}

Based on a two-step framework, this paper proposes a robust distributed control design for interconnected system under topology uncertainty. First, a set of distributed control laws is presented to ensure $H_{\infty}$ performance of system subject to topology uncertainty. By introducing a prespecified connectivity bound, an uncertainty set of topologies is characterized and the violation of stability condition can be determined locally. By minimizing $H_{2}$ norm of interconnected systems incorporating with communication penalty, we identify the optimal communication architecture from the admissible topology set. Additionally, an heuristic algorithm

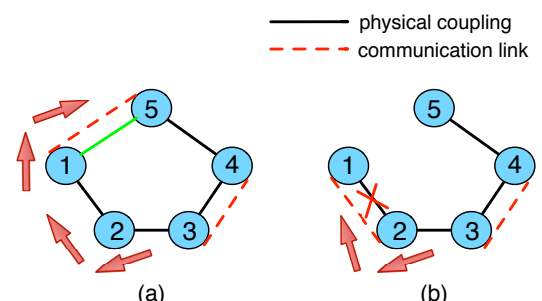

(a)

(b)

Fig. 3. manipulation of communication to deal with topology variations: (a). physical link $(1,5)$ added; (b). physical link $(1,2)$ lost

to reallocate the existing communication under topology variations is proposed by solving a suboptimal $H_{2}$ problem.

\section{ACKNOWLEDGMENTS}

This work is partially supported by the German Research Foundation (DFG) within the Priority Program SPP 1305 "Control Theory of Digitally Networked Dynamical Systems".

\section{REFERENCES}

[1] A. Kiani and A. Annaswamy, "Distributed hierarchical control for renewable energy integration in a smart grid," pp. 1-8, IEEE PES Innovative Smart Grid Technologies, 2012.

[2] A. Gusrialdi and S. Hirche, "On the explicit solution of communication topology design for distributed control of large-scale interconnected systems," pp. 6370-6375, American Control Conference, 2012.

[3] F. Lin, M. Fardad, and M. Jovanović, "Design of optimal sparse feedback gains via the alternating direction method of multipliers," Arxiv preprint arXiv: 1111.6188v2, 2011.

[4] M. Fardad, F. Lin, and M. Jovanović, "Sparsity-promoting optimal control for a class of distributed systems," pp. 2050-2055, American Control Conference, 2011.

[5] S. Schuler, P. Li, J. Lam, and F. Allgöwer, "Design of structured dynamic output-feedback controllers for interconnected systems," Internat. J. Control, vol. 84, pp. 2081-2091, 2011.

[6] A. Zečevič and D. Šiljak, Control of Complex Systems: Structural Constraints and Uncertainty. Boston: Springer, 2010.

[7] A. Gusrialdi and S. Hirche, "Performance-oriented communication topology design for large-scale interconnected systems," pp. 57075713, 49th IEEE Conference on Decision and Control, 2010.

[8] H. Ren and I. Dobson, "Using transmission line outage data to estimate cascading failure propagation in an electric power system," IEEE Trans. Circuits Syst. II, Exp. Briefs, vol. 55(9), pp. 927-931, 2008.

[9] F. Lin, M. Fardad, and M. R. Jovanović, "Augmented lagrangian approach to design of structured optimal state feedback gains," IEEE Trans. Auto. Cont, vol. 56(12), pp. 2923-2929, 2011.

[10] R. Cogill and S. Lall, "Topology independent control desing for networked systems," pp. 14-17, 43th IEEE Conference on Decision and Control, 2004.

[11] R. Cogill and S. Lall, "Control design for topology-independent stability of interconnected systems," pp. 3717-3722, American Control Conference, 2004.

[12] B. Korte and J. Vygen, Combinartorial Optimization: Theory and Algorithms. Springer, 2012.

[13] A. Ghosh and S. Boyd, "Growing well-connected graphs," pp. 66056611, 45th IEEE Conference on Decision and Control, 2006.

[14] J. Lofberg, "Yalmip: A toolbox for modelling and optimization in matlab," 2004.

[15] R. Tütüncü, K. Toh, and M. Todd, "Sdpt3 a matlab software package for semidefinite-quadraticlinear programming, version 3.0." Available: http://www.math.nus.edu.sg/mattohkc/. 